\title{
Anti-IL5 Drugs in COVID-19 Patients: Role of Eosinophils in SARS-CoV-2-Induced Immunopathology
}

\author{
Daniele Pala ${ }^{1,2 *}$ and Marco Pistis ${ }^{2,3}$ \\ ${ }^{1}$ Unit of Clinical Pharmacology, University Hospital Agency of Cagliari, Cagliari, Italy, ${ }^{2}$ Department of Biomedical Sciences, Division \\ of Neuroscience and Clinical Pharmacology, University of Cagliari, Cagliari, Italy, ${ }^{3}$ Neuroscience Institute, National Research \\ Council of Italy (CNR), Section of Cagliari, Cagliari, Italy
}

OPEN ACCESS

Edited by: Filippo Drago,

University of Catania, Italy

Reviewed by:

Mats W. Johansson,

University of Wisconsin-Madison,

United States

Rene Lutter,

University of Amsterdam, Netherlands

*Correspondence:

Daniele Pala

danielepala14@gmail.com

Specialty section:

This article was submitted to

Experimental Pharmacology and Drug

Discovery,

a section of the journal

Frontiers in Pharmacology

Received: 28 October 2020 Accepted: 03 February 2021

Published: 09 March 2021

Citation:

Pala D and Pistis M (2021) Anti-IL5 Drugs in COVID-19 Patients: Role of Eosinophils in SARS-CoV-2Induced Immunopathology. Front. Pharmacol. 12:622554. doi: 10.3389/fphar.2021.622554
SARS-CoV-2 infection stimulates a complex activation of the immune system. Eosinophils belong to the host's defense equipment against respiratory viruses. In the first phase of the infection, eosinophils contribution is probably appropriate and beneficial, as they facilitate the suppression of the viral replication. However, in severe COVID-19 patients, during the second and third phases of the disease, eosinophils may participate in a maladaptive immune response and directly contribute to immunopathology. In fact, in severe patients, the immune response is prevalently $T$ helper 1 type, but $T$ helper 2 is also present. Eosinophils' expansion and activation are stimulated by Type 2 cytokines, especially IL-5. Moreover, bronchial asthma, in which eosinophils play a central role, seems not to be a major risk factor for severe COVID-19. Among possible explanations, asthmatic patients are often treated with corticosteroids, which have been demonstrated to reduce the progression to critical COVID-19 in hospitalized patients. In addition to steroids, severe asthmatic patients are currently treated with biological drugs that target Type 2 immune response. Because IL-5 is necessary for the growth, survival, and activation of eosinophils, IL-5 inhibitors, such as mepolizumab, decrease the peripheral blood count of eosinophils, but do not influence eosinophils activation in the airway. In severe COVID-19 patients, the blockade of eosinophils' activation might contrast harmful immunity.

Keywords: COVID-19, asthma, type 2 response, eosinophils, interleukin-5, anti-IL5 drugs

\section{INTRODUCTION}

COVID-19 pandemic is a grave challenge for every health care professional worldwide. A better understanding of disease pathogenesis might boost a more effective and targeted therapy, with the hope of saving as many lives as possible. Here, a rationale for anti-IL5 drug use in severe COVID-19 patients is discussed. Since the beginning of the Health Emergency, several drugs, mostly antiviral and anti-inflammatory, have been repurposed and a vast number of clinical trials started worldwide to assess their efficacy and safety with an unprecedented speed. However, to date, only systemic corticosteroids have been demonstrated to prevent deaths in COVID-19 most severe patients and received formal approval from Regulatory Agencies (Sterne et al., 2020).

One of the most relevant findings regarding COVID-19's natural history highlights three different clinical stages, each requiring different types of therapies. In the first stage, the principal feature is 
viral replication, which can be contrasted by antiviral drugs, such as remdesivir. In the second stage, the pulmonary one, clinical symptoms become more prominent because of the beginning of the host's immune response. The third stage is characterized by an immunopathologic response, which can result in a cytokine storm in the most severe cases (Siddiqi and Mehra, 2020). In the second and third stages, immunotherapies can be indicated (Sandkovsky et al., 2020).

Mounting evidence shows that COVID-19's immunopathology has peculiar features. It has been highlighted that the classical $\mathrm{T}$ helper 1 (Th1) response is defective during acute infection, but it is prevalent among memory $\mathrm{T}$ cells in convalescent individuals (Sekine et al., 2020). Patients with moderate COVID-19 experience lower signs of inflammatory activation in comparison with severe COVID-19 patients. The inflammation follows an initial increment of cytokines, and subsequently a decrease of type 1 and type 3 responses (Lucas et al., 2020). The peripheral blood count of $\mathrm{CD}^{+}$and $\mathrm{CD} 8^{+} \mathrm{T}$ cells in terms of absolute number and frequency display a significant reduction in patients with either moderate or severe infection (Sekine et al., 2020). Nevertheless, Th1 $\mathrm{CD}^{+}$, Th1 $\mathrm{CD}^{+}$, and Natural Killer T cells are activated to promote antiviral activity and drive the disease recovery in moderate infection (Zhang J. Y. et al., 2020). Finally, in convalescent individuals, other authors analyzed SARS-CoV-2specific memory $\mathrm{T}$ cells and found that $\mathrm{CD} 4^{+} \mathrm{T}$ cells mainly produced Interferon- $\gamma$ (IFN- $\gamma$ ), Interleukin-2 (IL-2), and Tumor Necrosis Factor- $\alpha$ (TNF- $\alpha$ ), whereas $\mathrm{CD}^{+} \mathrm{T}$ cells mainly produced IFN- $\gamma$ (Sekine et al., 2020).

\section{TH2 IMMUNE RESPONSE IN SEVERE PATIENTS}

The first reports showed a significant difference in $\mathrm{T}$ helper 2 (Th2) cytokines in severe COVID-19 patients hospitalized into Intensive Care Units, especially IL-4 and IL-10, providing initial evidence in favor of Th2 activation, but significant differences in IL-5 levels were not found (Huang C. et al., 2020).

In severe patients, the immune system activation is characterized by a distorted interferon production and a disordered $\mathrm{T}$ cellular response that lead to profound immune exhaustion and broad T cell expansion (Zhang J. Y. et al., 2020). Patients with severe COVID-19 produce elevated levels of cytokines during the clinical course of the disease. These patients showed a pattern of Th1 activation, but also showed a Type 2 immune response, characterized by an increase of IL-5, IL-13, eotaxin-2, immunoglobulin E (IgE), and eosinophils. Type 2 biomarkers remain elevated in patients with severe COVID-19 and correlate with the worst course of the disease. Levels of eosinophils were significantly higher in patients with severe COVID-19 than those with a moderate disease or healthy controls (Lucas et al., 2020).

Similarly, levels of IL- 5 were higher in severe patients than in those with a moderate disease or healthy control. IL-13 differs in severe COVID-19 compared to controls. In patients with severe disease, IgE immunoglobulins slightly increased during the disease course (Lucas et al., 2020).

Conversely, IL-4 did not diverge between the two groups (Lucas et al., 2020). The last result is confirmed by another observational study (Mann et al., 2020). Nevertheless, IL-4, IL5 , and IL-13 displayed a trend toward an increase in the clinical scenario of severe COVID-19, and a reason for the interest is the importance of IL-5 to predict mortality, with a predictive value of around 0.73 (Lucas et al., 2020).

Elsewhere, eotaxins, a group of chemokines involved in the chemotaxis of eosinophils, revealed conflicting results: in the previous study, eotaxin-1 and eotaxin-3 were reduced in COVID19 (Lucas et al., 2020) similarly to another immunophenotyping study in which eotaxin-1 was reduced (Mathew et al., 2020). Differently, eotaxin-2 was increased as compared to controls (Lucas et al., 2020). The eosinophil count was similar and within the normal values range in the two studies: a mean count of 100 cells $/ \mu \mathrm{L}$ with a maximum of 250 cells $/ \mu \mathrm{L}$ in the first (Lucas et al., 2020) and count below 100 cells/ $\mu \mathrm{L}$ with a maximum at 400 cells $/ \mu \mathrm{L}$, in the second (Mathew et al., 2020). However, the two studies agree with the increase of fundamentals type 2 cytokines, like IL-5 and IL-13 in severe COVID-19 patients, at least in a subset of them (Lucas et al., 2020; Mathew et al., 2020). Another immunophenotyping study shows decreasing participation of eosinophils from mild to severe groups (Mann et al., 2020).

The specific activation of the type 2 immunity has been confirmed by different groups. Roncati et al. (2020) show that in all the 15 peripheral blood samples from intensive care COVID-19 patients, cytological signals of Th2 immune response were found, namely eosinophilia, basophilia, degranulated eosinophils, and plasma cells (Roncati et al., 2020). In general, stimulation of SARS-CoV-2-specific T cells from peripheral blood of severe COVID-19 patients drives a prevalent production of Th1 cytokines (IFN- $\gamma$, TNF- $\alpha$, IL-2), but also Th2 (IL-5, IL-13, IL-9, IL-10) and Th17 (IL-17A, IL-17F, and IL-22) cytokines were detected (Weiskopf et al., 2020). The specific T-cell response against SARS-CoV-2 was assessed by stimulating peripheral blood cells with the Spike protein and other viral peptides. Mononuclear blood cells from COVID-19 vs. non-COVID-19 cells produced a significant higher number of cytokines such as IL-2 (50.08 vs. 0), IFN- $\gamma$ (90.16 vs. 0), IL-4 (0.52 vs. 0$)$, IL-13 (0.84 vs. 0 ) and MCP-1 (4,602 vs 359.2), among which IL-4 and IL-13 are key Th2 mediators (Petrone et al., 2020). The involvement of IL- 4 and IL-13 was confirmed by others, who found a relative gene expression upregulation in $\mathrm{CD} 4^{+} \mathrm{T}$-cells from COVID-19 patients by using a single cell transcriptomics approach (Kalfaoglu et al., 2020).

In a single-cell analysis, six subtypes of $\mathrm{CD}^{+} \mathrm{T}$ cell clusters have been characterized. In particular, $2 \mathrm{~T} \mathrm{CD}^{+}$effector subtypes, CD4+-GZMK (granzyme) and CD4+-GNLY (granulysin) have been found. CD4+-GNLY cells displayed a high production of TBX21; consequently, they were Th1-like cells. Conversely, CD4+-GZMK and CD4+memory cells revealed Th2-like features with high production of GATA3 (Zhang J. Y. et al., 2020). 
To evaluate the differences between peripheral blood and bronchoalveolar lavage fluid (BALF) in COVID-19 patients, mononuclear cells from both compartments were compared. A clonal increase of Th1, Th2, and Th17 cells was found in severe cases. A significant difference has been observed between matched BALF and plasma samples for IL-5, IL-8, IL-17, and INF- $\alpha$, with higher levels of these cytokines in the BALF (Xu et al., 2020).

The timing analysis is a critical point to consider when observing the relative increase or decrease of a single cytokine or a cell subset. In a longitudinal analysis of a fatal case, IL-5 was found increased between 1- and 2-times on the 14th day since the infection, but showed a decrease between day 16 and day 22 and a further increase on day 24 (Bouadma et al., 2020). This pattern is in line with observations from a large-scale study that show an increase of IL-5 levels within days 6-11 from symptom onset, to which a subsequent increase of eosinophils follows on days 11-15, and a simultaneous slowdown of IL-5 rise on days 11-15. The last phase is characterised by a further increase of IL-5 on days 16-20 and a relative slowdown of blood eosinophil count on days 16-20 (Lucas et al., 2020). Finally, deceased patients had higher levels of IL-5 than patients with moderate or severe disease (Liu et al., 2020).

Probably, there are many explanations for these observations. In the first and early second phases of SARS-CoV-2 infection, eosinophils can contribute to the elimination of the virus, thanks to the antiviral activity of their enzymes. Later, during the advanced second phase of COVID-19, when the immune system starts slowing down viral replication, their antiviral properties are not requested, so IL-5 production is moderately reduced (Bouadma et al., 2020; Lucas et al., 2020). Nevertheless, eosinophils enrollment during the second phase may contribute to harm target tissues and progress the pathology. In the last phase of a severe course, the immune system undertakes a pathologic pathway characterized by a broad and uncontrolled cytokines storm, with a new pathological increase of IL-5 (Lucas et al., 2020; Bouadma et al., 2020; Liu et al., 2020).

\section{ROLE OF EOSINOPHILS IN PATHOLOGY}

Eosinophils play an important role in protecting the host against viral infections. They recognize viruses through Toll-like receptors (Flores-Torres et al., 2019). Eosinophils participate in the antiviral immune response because of their preformed granules, which contain cytotoxic proteins, such as eosinophil peroxidase, major basic protein, and 2 RNases (eosinophil neurotoxin and eosinophil cationic protein) (Ramirez et al., 2018; Flores-Torres et al., 2019). They produce reactive nitrogen species with antiviral activity (Flores-Torres et al., 2019).

Eosinophils protect the host from respiratory viruses, such as respiratory syncytial virus, rhinovirus, parainfluenza, and influenza virus (Ramirez et al., 2018). Eosinophils can rapidly internalize and inactivate respiratory syncytial virus and influenza virus, an ability that is compromised in asthma, with a close correlation with asthma exacerbation (Sabogal Piñeros et al., 2019a). Influenza A virus stimulates pulmonary lymphoid cells to generate large amounts of IL-5, which attracts eosinophils in the respiratory tissues (Gorski et al., 2019). In response to the influenza A virus, eosinophils activate, undergo degranulation, and act as antigen-presenting cells, then induce $\mathrm{CD}^{+} \mathrm{T}$ cell effector functions (Samarasinghe et al., 2017). A comprehensive review of eosinophils and viral infections was made by FloresTorres et al. (2019).

Toxic proteins and mediators released from activated eosinophils participate in the pathogenesis of asthma and other allergic and immune-mediated diseases (Ramirez et al., 2018). Asthma exacerbations can usually be triggered by viral infections (Flores-Torres et al., 2019). During asthma exacerbations, eosinophils are activated to release free eosinophil granules and undergo lysis (Muniz-Junqueira et al., 2013). In theory, two principal ways of dying exist for eosinophils: primary lysis and apoptosis. Surprisingly, some signals that induce eosinophils apoptosis, lead to cell lysis (Persson and Uller, 2013). Activated eosinophils from asthma and allergic diseases express on surface sialic acid-binding immunoglobulin-like lectin (Siglec)-8. Siglec-8 normally causes cell death, but in presence of IL-5, it induces ROS-dependent cell death, characterized by necrotic features and granules release (Kano et al., 2013). The characteristic of undergoing primary lysis clarify because apoptotic eosinophils have not been found yet in affected tissues from different eosinophilic diseases (Persson and Uller, 2013; Persson and Uller, 2014). Corticosteroids reduce eosinophilic granules in the sputum of asthma exacerbation and probably do anti-IL-5 drugs (Persson and Uller, 2014).

Primary lysis of eosinophils is characterised by cell membrane rupture, a subsequent release of free eosinophilic granules content, and damage-associated molecular patterns (DAMPs) from cytoplasm and nucleus (Persson and Uller, 2014). DAMPs, like ATP, High mobility group box 1 protein (HMGB1), RNAs, DNAs, and IL-1 $\beta$ stimulate a potent activation of inflammation. Through DAMPs signaling, a dying cell recruits phagocytes, like macrophages, dendritic cells, and epithelial cells. In turn, phagocytes, which have pattern recognition receptors (PRRs), start eating irreversibly damaged cells through a process called efferocytosis. Activated phagocytes by a necrotic dying cell produce pro-inflammatory cytokines such as IL-1, IL-6, and IL-12 (Kolb et al., 2017).

Interestingly, regarding COVID-19 pathology, phagocytes and related cytokines (especially IL-1 and IL-6) have been recognised to play a central role (Bonaventura et al., 2020). A similarity between COVID-19 and secondary Hemophagocytic lymphohistiocytosis (HLH) syndrome has been proposed (Mehta et al., 2020). HLH is characterized by ungoverned growth and activation of phagocytes. HLH is often triggered by viral infections, like herpes viruses (Epstein-Barr virus and cytomegalovirus mainly), H1N1 influenza virus, parvovirus B19, HIV, or other viruses. From a haematological point of view, HLH is characterized by leukopenia (Ramos-Casals et al., 2014).

A suggestive hypothesis is that, in COVID-19, eosinophils stimulated by SARS-CoV-2 infection would migrate to the lungs and undergo primary lysis, which in turn recruit phagocytes. In a severe patient, uncontrolled phagocyte activation causes hyper inflammation and a cytokine storm. The interplay between lung 
eosinophils and SARS-CoV-2 needs more in-depth analysis, considering potential therapeutic implications.

\section{EOSINOPHILS INVOLVEMENT IN COVID-19}

Since the first laboratory reports of COVID-19 severe patients, the peripheral blood count of circulating eosinophils is mostly found below the normal value range (Zhang Z. L. et al., 2020), and there is a significant difference between moderate vs. critical disease (Liao et al., 2020). These observations have been confirmed by many authors and by different meta-analyses (Danwang et al., 2020; Ghahramani et al., 2020).

Since the first phases of the infection, patients may undergo an active migration of circulating eosinophils from the peripheral blood to target tissues, because of their antiviral functions (FloresTorres et al., 2019). In the subsequent phases, peripheral eosinophils start declining. An explanation of the observed eosinopenia in the last phases of COVID-19 disease would consider the nearly concomitant increase of eosinophilsstimulating cytokines, such as IL-5 and GM-CSF, at least in a subset of patients (Lucas et al., 2020; Mathew et al., 2020), and the complex interactions with other actors of the immune system. A possible explanation is the induction of eosinophils apoptosis caused by endogenous or therapeutic glucocorticoids (Ilmarinen et al., 2014). Also, cytokines such as IFN- $\alpha$ and IFN- $\gamma$ (type 1 IFNs) can induce eosinophils apoptosis (Morita et al., 1996). Nevertheless, in COVID-19 type 1 IFNs production is limited (Acharya et al., 2020). Another explanation contemplates the induction of cell primary lysis (Persson and Uller, 2013). The last hypothesis has much more therapeutic implications because of the stimulation of efferocytosis and inflammation from primary lytic eosinophils (Persson and Uller, 2014).

During hospitalization, eosinophils', lymphocytes', and platelets' count showed a different pattern in the survivors' peripheral blood compared to the non-survivors: in survivors, the cell count increased progressively, whereas, in the nonsurvivor, it maintained low levels and finally declined. The laboratory eosinophils count is a negative prognostic factor for non-survivors, specifically eosinophils on hospital admission less than $0.03 \times 10^{9} / \mathrm{L}(\mathrm{HR}, 2.12 ; 95 \% \mathrm{CI}, 0.91-4.98)$, whereas eosinophils $\left(\mathrm{x} 10^{9} / \mathrm{L}\right)>0.05$ vs $\leq 0.05$ was a protective factor for fatal outcome (HR, 0.38; 95\% CI, 0.17-0.83). In Kaplan-Meier analysis, the survival was significantly higher in patients with eosinophils $>0.05\left(\mathrm{x} 10^{9} / \mathrm{L}\right)$ compared to those with eosinophils $\leq 0.05$ (x10 $/$ L) (Chen et al., 2020). Blood eosinophils showed a positive correlation with lymphocytes in severe and non-severe patients after admission (Zhang J. J. et al., 2020).

The laboratory monitoring of peripheral eosinophil count has been proposed as a precision tool to monitor the clinical course of the disease and predict the admission to Intensive Care Unit (ICU) (Huang J. et al., 2020). The eosinophils count $=0\left(\times 10^{9}\right.$ per L) predicts the admission to ICU with a mean sensitivity of 48.15 (95\% CI: 28.7-68.1) and a mean specificity of 98.88 (95\% CI: 93.9-100.0), a positive predictive value of 92.9 (95\% CI: 66.1-99.8) and a negative predictive value of 86.3 (95\% CI: 78.0-92.3). In the ROC analysis, the AUC of eosinophils is
0.763 (95\%CI: 0.641-0.886) (Sun et al., 2020). The very high value of specificity indicates that eosinophil might be a real target of the immune derangement like the decreased lymphocytes (Sun et al., 2020). Differently, the modest value of sensitivity might be explained by a certain degree of heterogeneity between severe COVID-19 patients, as demonstrated by immunophenotyping studies that identified different clusters of the cytokine storm signature (Lucas et al., 2020; Mathew et al., 2020). There is a statistically significant difference between peripheral eosinophils' blood count in non-severe and severe patients, pointing out that this population undergoes conditioning during the acute phase of severe infection (Sun et al., 2020; Xie et al., 2020).

Nevertheless, basophils and eosinophils should contribute to the antiviral response and could complicate the immunopathology. These cells undergo a dynamic change during severe disease: they increase from acute to recovery phases (Rodriguez et al., 2020). Other authors analyzed in more detail eosinophil cell populations. These populations exhibit a temporary growth of CD62L + eosinophils from day 2 to day 6 after hospital admission (Rodriguez et al., 2020). Expansion of CD62L + eosinophils seems to be attributed to IFN$\gamma$, one of the most relevant cytokines in severe COVID-19, and the IFN- $\gamma$ levels show an increase together with the increment of CD62L + eosinophils (Rodriguez et al., 2020). The specific phenotype of such eosinophils apparently belongs to a population of lung-resident eosinophils rather than to circulating eosinophils induced by the inflammation response, and CD62L + pulmonary-resident eosinophils have an important role in the organization of inflammatory responses in the lung (Mesnil et al., 2016). Rodriguez et al. (2020) think that the clonal growth of CD62L + eosinophils, which occurs after the development of a severe pulmonary immunopathology (one week after hospital admission) is correlated to the hyperinflammation of the lungs in COVID-19 patients (Rodriguez et al., 2020).

In a recent post-mortem series of SARS-CoV-2 deceased patients, eosinophils were found in the alveolar interstitium (Damiani et al., 2020). In a case report, pulmonary eosinophilic vasculitis (with transmural eosinophilic infiltrate) was found in a severe COVID-19 patient that underwent bronchopulmonary lavage and lung biopsy on day 32 after intubation. No allergic disorder was previously known. BALF showed $36 \%$ eosinophils and $2.4 \mathrm{pg} / \mathrm{ml}$ IL-5. After two weeks of corticosteroid treatment, a subsequent bronchoalveolar lavage was made that showed $3 \%$ eosinophils and $2.3 \mathrm{pg} / \mathrm{ml} \mathrm{IL-5} \mathrm{(Luecke}$ et al., 2021). Another case report described a clinical picture of eosinophilic pneumonia in a COVID-19 patient, diagnosed by increased eosinophils in BALF, which responded well to steroid treatment (Murao et al., 2020). However, it must be pointed out that the findings of eosinophils in severe COVID-19 lungs do not directly demonstrate that they are responsible for the damage. The role of eosinophils in pneumonia's immunopathology still needs to be fully understood. Another point favoring eosinophils' involvement is that skin dermatoses have been described in COVID-19 patients, in which increased eosinophils were found (Gianotti et al., 2020). The preferential expansion of lung-resident eosinophil is not in contrast with the 
observation that, in the most severe COVID-19 patients, peripheral blood count of eosinophils is generally decreased. Noteworthy, eosinopenia might depend on the migration of circulating eosinophils from the peripheral blood to the infected organs (Azkur et al., 2020).

\section{SEVERE ASTHMA IN COVID-19 PATIENTS: A CASE-STUDY}

Bronchial asthma is divided into two major phenotypes, which are characterized by Th2-high (eosinophilic) and Th2-low (noneosinophilic) immune responses (Kuruvilla et al., 2019). There is still a debate in the scientific literature if patients with bronchial asthma would be at increased risk of developing a severe COVID19 form and relative admission to the intensive care unit (Avdeev et al., 2020; Williamson et al., 2020; Choi H. G. et al., 2020). Until now, there are limited data about the effective risk of severe COVID-19 course in the population of asthmatic patients (Kow et al., 2020). A possible explanation because asthma does not appear to be a relevant risk factor for COVID-19 has been reported by Jackson et al. (2020) (Jackson et al., 2020). They hypothesized that atopic patients express lower levels of the ACE2 gene in their airways. In fact, SARS-CoV-2 uses the ACE2 receptor to infect the host's cells. Asthmatic children with allergen sensitization showed a progressive ACE2 decrease in the nasal epithelium. Similar results were reported in adults with mild asthma that received allergen provocation (Jackson et al., 2020).

Furthermore, a position paper from European Allergologists and Clinical Immunologists' leading societies highlights that there is currently no evidence for an increased risk of a severe COVID-19 course in allergic patients (Klimek et al., 2020). This statement is particularly surprising as asthma exacerbations can usually be triggered by respiratory infections (Flores-Torres et al., 2019). This interesting fact has been confirmed in different countries such as China, the USA, South Korea, and Italy (Klimek et al., 2020; Zhu et al., 2020). In detail, in Wuhan, the percentage of seriously ill or deceased COVID-19 patients with known bronchial asthma was far below the prevalence of asthma (Li et al., 2020). In a real-world observational study performed using administrative data from Korea, 7,590 confirmed SARSCoV-2 infection were identified. Among them, 218 (2.9\%) had asthma. The mortality rate was higher in asthmatic patients than non-asthmatic controls (7.8 vs. $2.8 \%$ ), but after adjusting for age, sex, and underlying conditions, asthma reveals not to be a significant risk factor for mortality (OR, 1.317; 95\% CI, 0.708-2.451). Indeed, none of the asthma treatments (including ICS, inhaled corticosteroids; LABA, long-acting $\beta 2$ agonists; LAMA, long-acting muscarinic antagonists; LTRA, leukotriene receptor antagonists; SABA, short-acting $\beta 2$ agonists) influences the mortality rate or admission to ICU in multivariate analysis and even asthma's severity was not associated with higher mortality (Choi Y. J. et al., 2020). These results were similar to those reported by another study from Daegu, Korea (Kim et al., 2020). Other authors demonstrated that asthma diagnosis was not associated with worse outcomes among severe COVID-19 patients 65 years or younger hospitalized within the New York City area, without considering age, obesity, or other high-risk comorbidities (Lovinsky-Desir et al., 2020). Other studies carried out in Italy confirm that the proportion of asthmatic patients in hospitalized COVID-19 positive patients is very small and suggest that asthma itself cannot be considered an independent risk factor for COVID 19 (Caminati et al., 2021) and does not appear to be one of the most relevant risk factors for ICU admission (Grasselli et al., 2020). There are several possible explanations for these findings. One explanation is that asthmatic patients are usually treated with systemic or inhaled corticosteroids, which have been demonstrated to ameliorate the disease course in severe COVID-19 (Peters et al., 2020; Rogliani et al., 2020; Sterne et al., 2020). Moreover, many severe asthmatic patients are under treatment with biologicals that inhibit type 2 immune responses via various mechanisms. The cited position paper does not dissuade to avoid such biological therapies but emphasizes that the potential effects of biologicals on the immune response in COVID-19 are currently unknown.

The first reports show that the disease course is not worse in COVID-19 patients with eosinophilic diseases under biological therapy, compared to those infected patients with eosinophilic disorder not treated with biologicals (Heffler et al., 2020). The pharmacological blockade of type 2 inflammation by therapeutic antibodies against IgE, IL-5, or IL-5/IL-4/-13 receptors, so far has not been suspected to increase the risk of viral infections, also in the respective approving clinical trials (Klimek et al., 2020). For example, in the Italian Severe Asthma Network (SANI) cohort, 26 patients received a confirmed (11) or a suspected diagnosis of COVID-19 (15). 21 patients with COVID-19 used biologicals: 15 (71\%) anti-IL-5 or anti-IL5R drugs (mepolizumab $\mathrm{n}=13$; benralizumab $\mathrm{n}=2$ ) and $6(29 \%)$ anti-IgE drug (omalizumab). In this small population, all patients were treated with inhaled corticosteroids/long-acting $\beta 2$-agonists, and only two patients deceased. The SANI registry cohort includes 1,504 patients, $65 \%$ of them receive biological treatments (anti-IL5 or anti-IL5R drugs: $52.9 \%$, anti-IgE: $47.1 \%$ ). In their large cohort of severe asthmatics, few COVID-19 diagnoses were made. The mortality rate among severe asthmatic patients with a SARS-CoV-2 diagnosis was $7.7 \%$, lower than that recorded in the Italian population (14.5\%). Among the severe asthmatic group with COVID-19, the majority were treated with anti-IL5 drugs (71\%), with a minority with anti-IgE (29\%) (Heffler et al., 2020). These preliminary data suggest that severe asthma subjects are not at higher risk of SARS-CoV-2 infection or development of a severe COVID-19 form of the disease.

Similar results were derived from the Belgian registry of Severe Asthma. Fourteen severe asthmatic patients with a SARS-CoV-2confirmed infection were collected from the registry cohort; five of them were hospitalized and none of them displayed asthma exacerbation, required systemic treatment with corticosteroids, invasive ventilation, or death. Only three patients received oxygen supplementation. Additionally, there was no difference in the incidence of SARS-CoV-2 infection between asthmatic patients who were on treatment with biologic therapy (four patients received anti-IgE and seven patients received anti-IL-5 or anti- 
IL-5R) and asthmatic patients not on treatment with biologics (Hanon et al., 2020). On the other hand, nine patients from the Dutch Severe Asthma Registry under biological treatment received a diagnosis of COVID-19, seven of them were hospitalized, and five entered to ICU. Six patients were on anti-IL5 therapy and three of them were admitted to ICU (all three patients were obese). Finally, only one patient died, but he had obesity and diabetes, known comorbidities for fatal outcome (Eger et al., 2020). Other case reports do not indicate a worse clinical course in COVID-19 patients exposed to antiIgE omalizumab (Lommatzsch et al., 2020) and, anti-IL-5R benralizumab (Renner et al., 2020a; Renner et al., 2020b). Although only a few cases were reported, it can be speculated that biological inhibitors of type 2 response can have a possible impact on aberrant immune response, and thus can protect infected subjects from severe complications of COVID-19.

Besides the potential protective effects of asthma medications, it must be pointed out that a possible explanation for a limited prevalence of asthma in COVID-19 patients might be because asthmatic patients are more aware of the greater risk of exacerbations of their condition, and thus have paid more attention to hygiene prescriptions and have been even more protected than the general population.

On the other hand, several reports challenge the hypothesis that severely asthmatic patients do not display a higher risk for severe COVID-19 (Choi H. G. et al., 2020; Williamson et al., 2020; Zhu et al., 2020); therefore, further studies focused on different asthma phenotypes are needed to better understand the association between asthma and COVID-19 severity.

\section{DISCUSSION}

To date, there are five monoclonal antibodies addressed against type 2 immune activation authorized as a specific target therapy for the treatment of severe eosinophilic asthma (Chaplin, 2020):

- omalizumab, which binds to IgE, blocking their interaction to the relative IgE receptor on basophils and mast cells; it downregulates the expression of IgE receptor (MacGlashan et al., 1997);

- benralizumab, an IL-5 receptor antagonist, which is expressed on the surface of eosinophils and basophils, provoking their apoptosis (Kolbeck et al., 2010);

- mepolizumab, targeted to IL-5, blocking the binding with the respective receptor expressed on eosinophils;

- reslizumab, targeted to IL-5, blocking the binding with the respective receptor expressed on eosinophils;

- dupilumab, which slows down type 2 inflammation, blocking IL-4 and IL-13;

The hypothesis presented here is based on the observation that in severe COVID-19 patients, Th2 immune response is stimulated and eosinophils may play a central role in precipitating immune derangement and aggravating SARSCoV-2-induced pneumonia. Because IL-5 is essential for the survival, maturation, and activation of eosinophils, it is suggested that IL-5 inhibitor drugs might block eosinophils activation in severe COVID-19 patients.

Immunophenotyping studies showed a moderate increase of eosinophils in severe COVID-19 patients, at least in a subset of them, with a mean blood count of around 100 cells $/ \mu \mathrm{L}$ (Lucas et al., 2020; Mathew et al., 2020). Anti-IL-5 drugs (benralizumab, mepolizumab, reslizumab) are currently indicated as an add-on therapy for subjects with severe eosinophilic asthma, not responding to standard treatments, diagnosed with a peripheral blood eosinophils count of 150 cells/ $\mu \mathrm{L}$ or higher at the beginning of treatment (GlaxoSmithKline, 2015; Teva, 2016; AstraZeneca, 2018). So, the question that arises is why such drugs should be beneficial in COVID-19 patients, especially those most severely affected.

In the EMA's Summary of Product Characteristic of mepolizumab, a combined analysis of the MEA112997 (DREAM) and MEA115588 (MENSA) approving trials is reported. Mepolizumab given at $75 \mathrm{mg}$ IV/100 mg s.c. provided a significant reduction rate of clinically asthma exacerbations when given to patients with severe refractory eosinophilic asthma with a baseline blood eosinophil count as low as $<150$ cells $/ \mu \mathrm{L}$, with a reduction of exacerbation rate of 0.67 . The effect size was larger, with an increasing count of eosinophils (GlaxoSmithKline, 2015). Mepolizumab reduces blood eosinophils and decreases the active migration of these cells to the lungs after stimulation with an allergen, but has a limited effect on respiratory resident eosinophils in asthma (Johansson et al., 2013; Kelly et al., 2017).

IL-5 receptor a (IL-5Ra) has been found in bronchial epithelial cells and allows epithelial barrier maintenance (Barretto et al., 2020). IL-5 beneficial effects on a mouse model of influenza do not depend only on eosinophils. Hence, IL-5Ra is expressed on migrated neutrophils in the lungs and neutrophils from other tissues (Gorski et al., 2019). IL-5Ra on activated neutrophils can promote signal transduction and, when activated by low concentrations of IL-5, causes a reduction of ROS production (Gorski et al., 2019). Children with asthma exacerbation exhibit both neutrophils and eosinophils recruitment and activation (Norzila et al., 2000). Interestingly, patients with mild asthma and rhinovirus infection that received mepolizumab treatment displayed a lower increase of neutrophils and neutrophil-derived myeloperoxidase in both BALF and sputum but also an increment of B lymphocytes and secretory IgA (Sabogal Piñeros et al., 2019b).

The role of neutrophils during SARS-CoV-2 infection is currently under investigation (Borges et al., 2020; Tomar et al., 2020; Wang et al., 2020). Specifically, the peripheral blood count of neutrophils is significantly higher in severe COVID-19 patients than those with moderate disease and can be considered a prognostic factor for a severe course (OR 1.5, 95\% CI: 1.0-2.1) (Wang et al., 2020). Moreover, neutrophils count increases within 7-9 days since symptoms onset and correlate with radiologic findings (Wang et al., 2020). Activated neutrophils drive the production of neutrophil extracellular traps (NET) composed of DNA and toxic proteins that lead to cell death (named NETosis) and tissue damage (Cheng and Palaniyar, 2013). Transcriptome analysis conducted in COVID-19 patients showed up-regulation 
of NET-associated genes. Thus, neutrophils and NETs can contribute to immunopathology in infected lungs (Wang et al., 2020). Anti-IL-5 therapy might block neutrophils' contribution to COVID-19 pneumonia.

IL-5R $\alpha$ has been found in B-cell progenitors and activated B cells. On B cells, IL-5 stimulation participates in the plasma cell differentiation process (Takatsu, 2011). However, mepolizumab promotes the activation of the antiviral immune response, like NK cells potentiation, B lymphocytes' survival, and IgA secretion (Contoli and Papi, 2019). Patients receiving mepolizumab should experience higher viral replication, so it should not be given in the first phase of the infection, like corticosteroids (Contoli and Papi, 2019).

Considering that eosinophils may participate in COVID-19's immunopathology and that anti-IL-5 drugs could be effective even starting from relatively modest levels of eosinophil counts, it is tempting to speculate that treating COVID-19 patients with such biologicals might prove beneficial. Because eosinophils participate in antiviral immune response, anti-IL-5 drugs should not be administered in patients in the first stage, characterised by viral replication and limited inflammation. In such a setting, these monoclonal antibodies might be detrimental, similarly to corticosteroids (Siddiqi and Mehra, 2020). High-risk patients with hypoxia in the second stage of the disease [namely pulmonary phase IIB according to Siddiqi et al. proposal (Siddiqi and Mehra, 2020)] might be the best setting to try using anti-IL-5 biologics and prevent eosinophils recruitment. This assumption is based on the observations made by Lucas et al. (2020), who showed an increase of IL-5 levels within 6-10 days from symptoms onset, and a subsequent increase of eosinophils count on 11-15 days from symptoms onset (Lucas et al., 2020). The clinical context is similar to that of the RECOVERY trial, whereby dexamethasone efficacy was demonstrated: namely patients who require oxygen therapy (from supplemental oxygen to mechanical ventilation) (Sterne et al., 2020; Horby et al., 2020). Consistently, the efficacy of dexamethasone on COVID-19 mortality maybe also due to eosinophils apoptosis induction (Ilmarinen et al., 2014).

It is predicted that hospitalised COVID-19 patients at risk of fatal outcome should be treated with anti-IL-5 drugs as soon as possible before peripheral eosinophil count falls. Clinical risk

\section{REFERENCES}

Acharya, D., Liu, G., and Gack, M. U. (2020). Dysregulation of type I interferon responses in COVID-19. Nat. Rev. Immunol. 20 (7), 397-398. doi:10.1038/ s41577-020-0346-x

AstraZeneca (2018). Fasenra $30 \mathrm{mg}$ solution for injection, summary of Product characteristics. Amsterdam, Netherlands: EMA.

Avdeev, S., Moiseev, S., Brovko, M., Yavorovskiy, A., Umbetova, K., Akulkina, L., et al. (2020). Low prevalence of bronchial asthma and chronic obstructive lung disease among intensive care unit patients with COVID-19. Allergy 75, 2703-2704. doi:10.1111/all.14420

Azkur, A. K., Akdis, M., Azkur, D., Sokolowska, M., van de Veen, W., Brüggen, M. C., et al. (2020). Immune response to SARS-CoV-2 and mechanisms of immunopathological changes in COVID-19. Allergy 75 (7), 1564-1581. doi:10.1111/all.14364 scores aiming to predict intensive care admission or death are still under investigation (Galloway et al., 2020), and the timing of changes in leucocyte counts (including that of eosinophils) is yet to be precisely determined in COVID-19.

An idea of the effect of IL-5 antagonism in severe COVID-19 may be inferred from the results of a small clinical trial that explored granulocyte-macrophage colony-stimulating factor (GM-CSF) antagonism (De Luca et al., 2020). GM-CSF is a growth factor produced by macrophages, T-cells, epithelial cells, endothelial cells, and fibroblasts: it promotes the survival of monocytes, the differentiation of macrophages, and the activation of $\mathrm{T}$ cells subpopulations (Bonaventura et al., 2020). GM-CSF facilitates the migration of eosinophils in the lung and promotes their survival, especially in a setting of allergic inflammation (Nobs et al., 2019). Mavrilimumab, a monoclonal antibody that blocks GM-CSF, has been shown to improve clinical outcomes compared to standard care in hospitalized patients (De Luca et al., 2020).

\section{CONCLUSION}

A reasoned timing and appropriate patient selection in a randomized controlled clinical trial is the only way to establish whether IL-5 antagonism in COVID-19 is beneficial or harmful. The second and early third stages of the disease, with high-risk moderate and severe patients, respectively, should be the appropriate setting to try using IL-5 drugs.

\section{AUTHOR CONTRIBUTIONS}

DP and MP wrote the manuscript.

\section{FUNDING}

This work was supported by "FSC 2014-2020-Patto per lo Sviluppo della Regione Sardegna, Legge Regionale n. 7 del 7 agosto 2007 (Bando 2017)" and by "Progetti di Rilevante Interesse Nazionale” (PRIN) 2017 (2017YH3SXK).

Barretto, K. T., Brockman-Schneider, R. A., Kuipers, I., Basnet, S., Bochkov, Y. A., Altman, M. C., et al. (2020). Human airway epithelial cells express a functional IL-5 receptor. Allergy 75 (8), 2127-2130. doi:10.1111/all.14297

Bonaventura, A., Vecchié, A., Wang, T. S., Lee, E., Cremer, P. C., Carey, B., et al. (2020). Targeting GM-CSF in COVID-19 pneumonia: rationale and strategies. Front. Immunol. 11, 1625. doi:10.3389/fimmu.2020.01625

Borges, L., Pithon-Curi, T. C., Curi, R., and Hatanaka, E. (2020). COVID-19 and neutrophils: the relationship between hyperinflammation and neutrophil extracellular traps. Mediators Inflamm. 2020, 8829674. doi:10.1155/2020/ 8829674

Bouadma, L., Wiedemann, A., Patrier, J., Surénaud, M., Wicky, P. H., Foucat, E., et al. (2020). Immune alterations in a patient with SARS-CoV-2-related acute respiratory distress syndrome. J. Clin. Immunol. 40 (8), 1082-1092. doi:10. 1007/s10875-020-00839-x

Caminati, M., Vultaggio, A., Matucci, A., Senna, G., Almerigogna, F., Bagnasco, D., et al. (2021). Asthma in a large COVID-19 cohort: prevalence, features, and 
determinants of COVID-19 disease severity. Respir. Med. 176, 106261. doi:10. 1016/j.rmed.2020.106261

Chaplin, S. (2020). Monoclonal antibodies for the treatment of severe asthma. Prescriber 31 (3), 23-28. doi:10.1002/psb.1830

Chen, R., Sang, L., Jiang, M., Yang, Z., Jia, N., Fu, W., et al. (2020). Longitudinal hematologic and immunologic variations associated with the progression of COVID-19 patients in China. J. Allergy Clin. Immunol. 146 (1), 89-100. doi:10. 1016/j.jaci.2020.05.003

Cheng, O., and Palaniyar, N. (2013). NET balancing: a problem in inflammatory lung diseases. Front. Immunol. 4, 1. doi:10.3389/fimmu.2013.00001

Choi, H. G., Wee, J. H., Kim, S. Y., Kim, J. H., Kim, H. I., Park, J. Y., et al. (2020). Association between asthma and clinical mortality/morbidity in COVID-19 patients using clinical epidemiologic data from Korean Disease Control and Prevention. Allergy. doi:10.1111/all.14675

Choi, Y. J., Park, J. Y., Lee, H. S., Suh, J., Song, J. Y., Byun, M. K., et al. (2020). Effect of asthma and asthma medication on the prognosis of patients with COVID-19. Eur. Respir. J. 2002226. doi:10.1183/13993003.02226-2020

Contoli, M., and Papi, A. (2019). Effects of anti-IL-5 on virus-induced exacerbation in asthma. Light and shadow. Am. J. Respir. Crit. Care Med. 199 (4), 410-411. doi:10.1164/rccm.201809-1684ED

Damiani, S., Fiorentino, M., de Palma, A., Foschini, M. P., Lazzarotto, T., Gabrielli, L., et al. (2020). Pathological post mortem findings in lungs infected with sarscov 2. J. Pathol. 253, 31-40. doi:10.1002/path.5549

Danwang, C., Endomba, F. T., Nkeck, J. R., Wouna, D. L. A., Robert, A., and Noubiap, J. J. (2020). A meta-analysis of potential biomarkers associated with severity of coronavirus disease 2019 (COVID-19). Biomark Res. 8, 37. doi:10. 1186/s40364-020-00217-0

De Luca, G., Cavalli, G., Campochiaro, C., Della-Torre, E., Angelillo, P., Tomelleri, A., et al. (2020). GM-CSF blockade with mavrilimumab in severe COVID-19 pneumonia and systemic hyperinflammation: a single-centre, prospective cohort study. Lancet Rheumatol. 2 (8), e465-e473. doi:10.1016/S26659913(20)30170-3

Eger, K., Hashimoto, S., Braunstahl, G. J., Brinke, A. T., Patberg, K. W., Beukert, A., et al. (2020). Poor outcome of SARS-CoV-2 infection in patients with severe asthma on biologic therapy. Respir. Med. 177, 106287. doi:10.1016/j.rmed.2020. 106287

Flores-Torres, A. S., Salinas-Carmona, M. C., Salinas, E., and Rosas-Taraco, A. G. (2019). Eosinophils and respiratory viruses. Viral Immunol. 32 (5), 198-207. doi:10.1089/vim.2018.0150

Galloway, J. B., Norton, S., Barker, R. D., Brookes, A., Carey, I., Clarke, B. D., et al. (2020). A clinical risk score to identify patients with COVID-19 at high risk of critical care admission or death: an observational cohort study. J. Infect. 81 (2), 282-288. doi:10.1016/j.jinf.2020.05.064

Ghahramani, S., Tabrizi, R., Lankarani, K. B., Kashani, S. M. A., Rezaei, S., Zeidi, N., et al. (2020). Laboratory features of severe vs. non-severe COVID-19 patients in Asian populations: a systematic review and meta-analysis. Eur. J. Med. Res. 25 (1), 30. doi:10.1186/s40001-020-00432-3

Gianotti, R., Recalcati, S., Fantini, F., Riva, C., Milani, M., Dainese, E., et al. (2020). Histopathological study of a broad spectrum of skin dermatoses in patients affected or highly suspected of infection by COVID-19 in the northern part of Italy: analysis of the many faces of the viral-induced skin diseases in previous and new reported cases. Am. J. Dermatopathol. 42 (8), 564-570. doi:10.1097/ DAD. 0000000000001707

GlaxoSmithKline, Nucala. (2015). Nucala 100 mg solution for injection, summary of Product characteristics. Amsterdam, Netherlands: EMA.

Gorski, S. A., Lawrence, M. G., Hinkelman, A., Spano, M. M., Steinke, J. W., Borish, L., et al. (2019). Expression of IL-5 receptor alpha by murine and human lung neutrophils. PloS one 14 (8), e0221113. doi:10.1371/journal. pone. 0221113

Grasselli, G., Zangrillo, A., Zanella, A., Antonelli, M., Cabrini, L., Castelli, A., et al. (2020). Baseline characteristics and outcomes of 1591 patients infected with SARS-CoV-2 admitted to ICUs of the lombardy region, Italy. JAMA 323 (16), 1574-1581. doi:10.1001/jama.2020.5394

Hanon, S., Brusselle, G., Deschampheleire, M., Louis, R., Michils, A., Peché, R., et al. (2020). COVID-19 and biologics in severe asthma: data from the belgian severe asthma registry. Eur. Respir. J. 56, 2002847. doi:10.1183/13993003. 02857-2020
Heffler, E., Detoraki, A., Contoli, M., Papi, A., Paoletti, G., Malipiero, G., et al. (2020). COVID-19 in Severe Asthma Network in Italy (SANI) patients: clinical features, impact of comorbidities and treatments. Allergy. doi:10.1111/all.14532

Horby, P., Lim, W. S., Emberson, J. R., Mafham, M., Bell, J. L., Linsell, L., et al. (2020). Dexamethasone in hospitalized patients with covid-19-preliminary report. N. Engl. J. Med. doi:10.1056/NEJMoa2021436

Huang, C., Wang, Y., Li, X., Ren, L., Zhao, J., Hu, Y., et al. (2020). Clinical features of patients infected with 2019 novel coronavirus in Wuhan, China. Lancet 395 (10223), 497-506. doi:10.1016/S0140-6736(20)30183-5

Huang, J., Zhang, Z., Liu, S., Gong, C., Chen, L., Ai, G., et al. (2020). Absolute eosinophil count predicts intensive care unit transfer among elderly COVID-19 patients from general isolation wards. Front. Med. 7, 585222. doi:10.3389/fmed. 2020.585222

Ilmarinen, P., Moilanen, E., and Kankaanranta, H. (2014). Regulation of spontaneous eosinophil apoptosis-a neglected area of importance. J. Cel Death 7, 1-9. doi:10.4137/JCD.S13588

Jackson, D. J., Busse, W. W., Bacharier, L. B., Kattan, M., O'Connor, G. T., Wood, R. A., et al. (2020). Association of respiratory allergy, asthma, and expression of the SARS-CoV-2 receptor ACE2. J. Allergy Clin. Immunol. 146 (1), 203-206.e3. doi:10.1016/j.jaci.2020.04.009

Johansson, M. W., Gunderson, K. A., Kelly, E. A., Denlinger, L. C., Jarjour, N. N., and Mosher, D. F. (2013). Anti-IL-5 attenuates activation and surface density of $\beta(2)$-integrins on circulating eosinophils after segmental antigen challenge. Clin. Exp. Allergy 43 (3), 292-303. doi:10.1111/j.1365-2222.2012.04065.x

Kalfaoglu, B., Almeida-Santos, J., Tye, C. A., Satou, Y., and Ono, M. (2020). T-cell hyperactivation and paralysis in severe COVID-19 infection revealed by singlecell analysis. Front. Immunol. 11, 589380. doi:10.3389/fimmu.2020.589380

Kano, G., Almanan, M., Bochner, B. S., and Zimmermann, N. (2013). Mechanism of Siglec-8-mediated cell death in IL-5-activated eosinophils: role for reactive oxygen species-enhanced MEK/ERK activation. J. Allergy Clin. Immunol. 132 (2), 437-445. doi:10.1016/j.jaci.2013.03.024

Kelly, E. A., Esnault, S., Liu, L. Y., Evans, M. D., Johansson, M. W., Mathur, S., et al. (2017). Mepolizumab attenuates airway eosinophil numbers, but not their functional phenotype in asthma. Am. J. Respir. Crit. Care Med. 196 (11), 1385-1395. doi:10.1164/rccm.201611-2234OC

Kim, S., Jung, C. G., Lee, J. Y., Kim, G., Choi, S. W., Jin, H. J., et al. (2020). Characterization of asthma and risk factors for delayed SARS-CoV-2 clearance in adult COVID-19 inpatients in Daegu. Allergy. doi:10.1111/all.14609

Klimek, L., Pfaar, O., Worm, M., Eiwegger, T., Hagemann, J., Ollert, M., et al. (2020). Use of biologicals in allergic and type-2 inflammatory diseases during the current COVID-19 pandemic: position paper of Ärzteverband Deutscher Allergologen (AeDA)(A), Deutsche Gesellschaft für Allergologie und Klinische Immunologie (DGAKI)(B), Gesellschaft für Pädiatrische Allergologie und Umweltmedizin (GPA)(C), Österreichische Gesellschaft für Allergologie und Immunologie (ÖGAI)(D), Luxemburgische Gesellschaft für Allergologie und Immunologie (LGAI)(E), Österreichische Gesellschaft für Pneumologie (ÖGP)(F) in co-operation with the German, Austrian, and Swiss ARIA groups(G), and the European Academy of Allergy and Clinical Immunology (EAACI)(H). Allergol. Select 4, 53-68. doi:10.5414/ALX02166E

Kolb, J. P., Oguin, T. H., Oberst, A., and Martinez, J. (2017). Programmed cell death and inflammation: winter is coming. Trends Immunol. 38 (10), 705-718. doi:10. 1016/j.it.2017.06.009

Kolbeck, R., Kozhich, A., Koike, M., Peng, L., Andersson, C. K., Damschroder, M. M., et al. (2010). MEDI-563, a humanized anti-IL-5 receptor alpha mAb with enhanced antibody-dependent cell-mediated cytotoxicity function. J. Allergy Clin. Immunol. 125 (6), 1344-e2. doi:10.1016/j.jaci.2010.04.004

Kow, C. S., Capstick, T., and Hasan, S. S. (2020). Are severe asthma patients at higher risk of developing severe outcomes from COVID-19? Allergy doi:10. 1111/all.14589

Kuruvilla, M. E., Lee, F. E.-H., and Lee, G. B. (2019). Understanding asthma phenotypes, endotypes, and mechanisms of disease. Clin. Rev. Allergy Immunol. 56 (2), 219-233. doi:10.1007/s12016-018-8712-1

Li, X., Xu, S., Yu, M., Wang, K., Tao, Y., Zhou, Y., et al. (2020). Risk factors for severity and mortality in adult COVID-19 inpatients in Wuhan. J. Allergy Clin. Immunol. 146 (1), 110-118. doi:10.1016/j.jaci.2020.04.006

Liao, D., Zhou, F., Luo, L., Xu, M., Wang, H., Xia, J., et al. (2020). Haematological characteristics and risk factors in the classification and prognosis evaluation of 
COVID-19: a retrospective cohort study. Lancet Haematol. 7 (9), e671-e678. doi:10.1016/S2352-3026(20)30217-9

Liu, Y., Chen, D., Hou, J., Li, H., Cao, D., Guo, M., et al. (2020). An inter-correlated cytokine network identified at the center of cytokine storm predicted COVID19 prognosis. Cytokine, 155365. doi:10.1016/j.cyto.2020.155365

Lommatzsch, M., Stoll, P., and Virchow, J. C. (2020). COVID-19 in a patient with severe asthma treated with omalizumab. Allergy 75, 2705-2708. doi:10.1111/all. 14456

Lovinsky-Desir, S., Deshpande, D. R., De, A., Murray, L., Stingone, J. A., Chan, A., et al. (2020). Asthma among hospitalized patients with COVID-19 and related outcomes. J. Allergy Clin. Immunol. 146, 1027-1034.e4. doi:10.1016/j.jaci.2020. 07.026

Lucas, C., Wong, P., Klein, J., Castro, T. B. R., Silva, J., Sundaram, M., et al. (2020). Longitudinal analyses reveal immunological misfiring in severe COVID-19. Nature 584 (7821), 463-469. doi:10.1038/s41586-020-2588-y

Luecke, E., Jeron, A., Kroeger, A., Bruder, D., Stegemann-Koniszewski, S., Jechorek, D., et al. (2021). Eosinophilic pulmonary vasculitis as a manifestation of the hyperinflammatory phase of COVID-19. J. Allergy Clin. Immunol. 147 (1), 112-113. doi:10.1016/j.jaci.2020.09.026

MacGlashan, D. W., Jr., Bochner, B. S., Adelman, D. C., Jardieu, P. M., Togias, A., McKenzie-White, J., et al. (1997). Down-regulation of Fc(epsilon)RI expression on human basophils during in vivo treatment of atopic patients with anti-IgE antibody. J. Immunol. 158 (3), 1438-1445.

Mann, E. R., Menon, M., Knight, S. B., Konkel, J. E., Jagger, C., Shaw, T. N., et al. (2020). Longitudinal immune profiling reveals key myeloid signatures associated with COVID-19. Sci. Immunol. 5 (51). doi:10.1126/sciimmunol. abd6197

Mathew, D., Giles, J. R., Baxter, A. E., Oldridge, D. A., Greenplate, A. R., Wu, J. E., et al. (2020). Deep immune profiling of COVID-19 patients reveals distinct immunotypes with therapeutic implications. Science 369 (6508). doi:10.1126/ science.abc 8511

Mehta, P., McAuley, D. F., Brown, M., Sanchez, E., Tattersall, R. S., and Manson, J. J. (2020). COVID-19: consider cytokine storm syndromes and immunosuppression. The Lancet 395 (10229), 1033-1034. doi:10.1016/ s0140-6736(20)30628-0

Mesnil, C., Raulier, S., Paulissen, G., Xiao, X., Birrell, M. A., Pirottin, D., et al. (2016). Lung-resident eosinophils represent a distinct regulatory eosinophil subset. J. Clin. Invest. 126 (9), 3279-3295. doi:10.1172/JCI85664

Morita, M., Lamkhioued, B., Soussi Gounni, A., Aldebert, D., Delaporte, E., Capron, A., et al. (1996). Induction by interferons of human eosinophil apoptosis and regulation by interleukin-3, granulocyte/macrophage-colony stimulating factor and interleukin-5. Eur. Cytokine Netw. 7 (4), 725-732.

Muniz-Junqueira, M. I., Barbosa-Marques, S. M., and Junqueira, L. F., Jr. (2013). Morphological changes in eosinophils are reliable markers of the severity of an acute asthma exacerbation in children. Allergy 68 (7), 911-920. doi:10.1111/all. 12176

Murao, K., Saito, A., Kuronuma, K., Fujiya, Y., Takahashi, S., and Chiba, H. (2020). Acute eosinophilic pneumonia accompanied with COVID-19: a case report. Respirol. Case Rep. 8 (9), e00683. doi:10.1002/rcr2.683

Nobs, S. P., Kayhan, M., and Kopf, M. (2019). GM-CSF intrinsically controls eosinophil accumulation in the setting of allergic airway inflammation. J. Allergy Clin. Immunol. 143 (4), 1513-1524. doi:10.1016/j.jaci.2018.08.044

Norzila, M. Z., Fakes, K., Henry, R. L., Simpson, J., and Gibson, P. G. (2000). Interleukin-8 secretion and neutrophil recruitment accompanies induced sputum eosinophil activation in children with acute asthma. Am. J. Respir. Crit. Care Med. 161 (3), 769-774. doi:10.1164/ajrccm.161.3.9809071

Persson, C., and Uller, L. (2013). Primary lysis of eosinophils as a major mode of activation of eosinophils in human diseased tissues. Nat. Rev. Immunol. 13 (12), 902. doi:10.1038/nri3341-c1

Persson, C., and Uller, L. (2014). Theirs but to die and do: primary lysis of eosinophils and free eosinophil granules in asthma. Am. J. Respir. Crit. Care Med. 189 (6), 628-633. doi:10.1164/rccm.201311-2069OE

Peters, M. C., Sajuthi, S., Deford, P., Christenson, S., Rios, C. L., Montgomery, M. T., et al. (2020). COVID-19-related genes in sputum cells in asthma. Relationship to demographic features and corticosteroids. Am. J. Respir. Crit. Care Med. 202 (1), 83-90. doi:10.1164/rccm.202003-0821OC

Petrone, L., Petruccioli, E., Vanini, V., Cuzzi, G., Fard, S. N., Alonzi, T., et al. (2020). A whole blood test to measure SARS-CoV-2-specific response in COVID-19 patients. Clin. Microbiol. Infect. 27, 286.e7-286.e13. doi:10.1016/j.cmi.2020. 09.051

Ramirez, G. A., Yacoub, M. R., Ripa, M., Mannina, D., Cariddi, A., Saporiti, N., et al. (2018). Eosinophils from physiology to disease: a comprehensive review. Biomed. Res. Int. 2018, 9095275. doi:10.1155/2018/9095275

Ramos-Casals, M., Brito-Zerón, P., López-Guillermo, A., Khamashta, M. A., and Bosch, X. (2014). Adult haemophagocytic syndrome. Lancet 383 (9927), 1503-1516. doi:10.1016/S0140-6736(13)61048-X

Renner, A., Marth, K., Patocka, K., Idzko, M., and Pohl, W. (2020a). COVID-19 in two severe asthmatics receiving benralizumab: busting the eosinophilia myth. ERJ Open Res. 6 (4). doi:10.1183/23120541.00457-2020

Renner, A., Marth, K., Patocka, K., and Pohl, W. (2020b). COVID-19 in a severe eosinophilic asthmatic receiving benralizumab-a case study. J. Asthma, 1-3. doi:10.1080/02770903.2020.1781165

Rodriguez, L., Pekkarinen, P. T., Lakshmikanth, T., Tan, Z., Consiglio, C. R., Pou, C., et al. (2020). Systems-level immunomonitoring from acute to recovery phase of severe COVID-19. Cell Rep. Med. 1 (5), 100078. doi:10.1016/j.xcrm.2020. 100078

Rogliani, P., Lauro, D., Di Daniele, N., Chetta, A., and Calzetta, L. (2020). Reduced risk of COVID-19 hospitalization in asthmatic and COPD patients: a benefit of inhaled corticosteroids? Expert Rev. Respir. Med., 1-8. doi:10.1080/17476348. 2021.1850275

Roncati, L., Nasillo, V., Lusenti, B., and Riva, G. (2020). Signals of Th2 immune response from COVID-19 patients requiring intensive care. Ann. Hematol. 99 (6), 1419-1420. doi:10.1007/s00277-020-04066-7

Sabogal Piñeros, Y. S., Bal, S. M., Dijkhuis, A., Majoor, C. J., Dierdorp, B. S., Dekker, T., et al. (2019a). Eosinophils capture viruses, a capacity that is defective in asthma. Allergy 74 (10), 1898-1909. doi:10.1111/all.13802

Sabogal Piñeros, Y. S., Bal, S. M., van de Pol, M. A., Dierdorp, B. S., Dekker, T., Dijkhuis, A., et al. (2019b). Anti-IL-5 in mild asthma alters rhinovirus-induced macrophage, B-cell, and neutrophil responses (material). A placebo-controlled, double-blind study. Am. J. Respir. Crit. Care Med. 199 (4), 508-517. doi:10. 1164/rccm.201803-0461oc

Samarasinghe, A. E., Melo, R. C., Duan, S., LeMessurier, K. S., Liedmann, S., Surman, S. L., et al. (2017). Eosinophils promote antiviral immunity in mice infected with influenza A virus. J. Immunol. 198 (8), 3214. doi:10.4049/ jimmunol.1600787

Sandkovsky, U., Colley, P., Sam, T., Modrykamien, A. M., and Berhe, M. (2020). The dynamic treatment of SARS-CoV-2 disease. Proc (Bayl. Univ. Med. Cent.) 33 (4), 572-579. doi:10.1080/08998280.2020.1780086

Sekine, T., Perez-Potti, A., Rivera-Ballesteros, O., Strålin, K., Gorin, J. B., Olsson, A., et al. (2020). Robust $\mathrm{T}$ cell immunity in convalescent individuals with asymptomatic or mild COVID-19. Cell 183 (1), 158-168.e14. doi:10.1016/j.cell. 2020.08.017

Siddiqi, H. K., and Mehra, M. R. (2020). COVID-19 illness in native and immunosuppressed states: a clinical-therapeutic staging proposal. J. Heart Lung Transplant. 39 (5), 405-407. doi:10.1016/j.healun.2020.03.012

Sterne, J. A. C., Murthy, S., Diaz, J. V., Slutsky, A. S., Villar, J., Angus, D. C., et al. (2020). Association between administration of systemic corticosteroids and mortality among critically ill patients with COVID-19: a meta-analysis. JAMA 324, 1330-1341. doi:10.1001/jama.2020.17023

Sun, S., Cai, X., Wang, H., He, G., Lin, Y., Lu, B., et al. (2020). Abnormalities of peripheral blood system in patients with COVID-19 in Wenzhou, China. Clin. Chim. Acta 507, 174-180. doi:10.1016/j.cca.2020.04.024

Takatsu, K. (2011). Interleukin-5 and IL-5 receptor in health and diseases. Proc. Jpn. Acad. Ser. B, Phys. Biol. Sci. 87 (8), 463-485. doi:10.2183/pjab.87.463

Teva (2016). Cinqaero $10 \mathrm{mg} / \mathrm{mL}$ concentrate for solution for infusion, Summary of Product Characteristics. Amsterdam, Netherlands: EMA.

Tomar, B., Anders, H. J., Desai, J., and Mulay, S. R. (2020). Neutrophils and neutrophil extracellular traps drive necroinflammation in COVID-19. Cells 9 (6). doi:10.3390/cells 9061383

Wang, J., Li, Q., Yin, Y., Zhang, Y., Cao, Y., Lin, X., et al. (2020). Excessive neutrophils and neutrophil extracellular traps in COVID-19. Front. Immunol. 11, 2063. doi:10.3389/fimmu.2020.02063

Weiskopf, D., Schmitz, K. S., Raadsen, M. P., Grifoni, A., Okba, N. M. A., Endeman, H., et al. (2020). Phenotype and kinetics of SARS-CoV-2-specific T cells in COVID-19 patients with acute respiratory distress syndrome. Sci. Immunol. 5 (48), eabd2071. doi:10.1126/sciimmunol.abd2071 
Williamson, E. J., Walker, A. J., Bhaskaran, K., Bacon, S., Bates, C., Morton, C. E., et al. (2020). Factors associated with COVID-19-related death using OpenSAFELY. Nature 584 (7821), 430-436. doi:10.1038/s41586-0202521-4

Xie, G., Ding, F., Han, L., Yin, D., Lu, H., and Zhang, M. (2020). The role of peripheral blood eosinophil counts in COVID-19 patients. Allergy 76, 471-482. doi:10.1111/all.14465

Xu, G., Qi, F., Li, H., Yang, Q., Wang, H., Wang, X., et al. (2020). The differential immune responses to COVID-19 in peripheral and lung revealed by single-cell RNA sequencing. Cell Discov. 6, 73. doi:10.1038/ s41421-020-00225-2

Zhang, J. J., Dong, X., Cao, Y. Y., Yuan, Y. D., Yang, Y. B., Yan, Y. Q., et al. (2020). Clinical characteristics of 140 patients infected with SARS-CoV-2 in Wuhan, China. Allergy 75 (7), 1730-1741. doi:10.1111/all.14238

Zhang, J. Y., Wang, X. M., Xing, X., Xu, Z., Zhang, C., Song, J. W., et al. (2020). Single-cell landscape of immunological responses in patients with COVID-19. Nat. Immunol. 21 (9), 1107-1118. doi:10.1038/s41590-0200762-x
Zhang, Z. L., Hou, Y. L., Li, D. T., and Li, F. Z. (2020). Laboratory findings of COVID-19: a systematic review and meta-analysis. Scand. J. Clin. Lab. Invest. 80 (6), 441-447. doi:10.1080/00365513.2020.1768587

Zhu, Z., Hasegawa, K., Ma, B., Fujiogi, M., Camargo, C. A., and Liang, L. (2020). Association of asthma and its genetic predisposition with the risk of severe COVID-19. J. Allergy Clin. Immunol. 146 (2), 327-329.e4. doi:10.1016/j.jaci. 2020.06.001

Conflict of Interest: The authors declare that the research was conducted in the absence of any commercial or financial relationships that could be construed as a potential conflict of interest.

Copyright $(2021$ Pala and Pistis. This is an open-access article distributed under the terms of the Creative Commons Attribution License (CC BY). The use, distribution or reproduction in other forums is permitted, provided the original author(s) and the copyright owner(s) are credited and that the original publication in this journal is cited, in accordance with accepted academic practice. No use, distribution or reproduction is permitted which does not comply with these terms. 